\title{
A Liquid-Helium Cold Cell for Use With an X-ray Diffractometer
}

\author{
Igor A. Black, ${ }^{1}$ Leonard H. Bolz, Frank P. Brooks, ${ }^{1}$ Floyd A. Mauer, and H. Steffen Peiser
}

A liquid-helium cold cell for use with the General Electric X-ray goniometer has been designed and constructed. It is used to obtain X-ray diffraction patterns of polverystalline solids deposited from the gaseous phase on a helium-cooled surface. This work is part of the program for studying the stabilization of free radicals.

\section{Introduction}

A 3-yr program of basic research has been undertaken at the National Bureau of Standards to study the preparation of free radicals and the preservation of these unstable molecular fragments by freezing. The usual technique involves dissociating a gas in a furnace, an rf field, or a spark discharge, and depositing the products on a surface cooled by liquid helium.

The freezing technique is widely used to store molecular fragments for study by spectroscopy, calorimetry, paramagnetic resonance, and other techniques. A knowledge of the structures of the solids deposited and the transformations they undergo during warmup is important in interpreting the results of these experiments. In calorimetry, for example, it may be difficult to determine whether an observed heat effect should be attributed to recombination of free radicals or crystallization of an amorphous deposit. To aid in settling such questions, a study of the structures and transformations of solids encountered in free-radical research was initiated. Special equipment was used for freezing gaseous samples at liquid-helium temperature and obtaining X-ray powder diffraction patterns. This paper describes the construction and use of the liquid-helium cold cell or cryostat developed for this work.

\section{Selection of Techniques and Equipment}

Reviews of the literature on low-temperature Xray diffraction by Ruhemann $[1]^{2}$ and by Steward [2] as well as the additional references at the end of this paper [3 to 8] show that a considerable number of X-ray crvostats have been described. Most of these, however, are for use with film cameras and are not well adapted to observing transient effects that occur on warmup of samples containing trapped free radicals. Of the diffractometer cryostats described, only the one by Barrett [7] can be used at liquid-helium temperature, and it is designed to fit $\mathrm{X}$-ray equipment that is no longer available.

In order to take advantage of the rapid-recording feature of modern electronic-counter diffractometers, design of a cryostat that could be substituted for the room-temperature specimen mount on one of

Arthur D. Little, Inc

Figures in brackets indicate the literature reference at the end of this paper. these instruments was undertaken. The X-ray equipment selected for modification was the General Electric diffractometer, the advantage of this instrument being that it has a vertical goniometer axis. The cryostat can be made to turn on this axis as required without causing liquid refrigerants to shift position.

\section{Design and Construction}

Once the X-ray technique and equipment had been selected, design of the cryostat followed along fairly conventional lines. It may be considered to have evolved from the optical cold cell described by Duerig and Mador [9]. X-ray windows and other special fittings had to be provided, and many changes were made to take advantage of modern manufacturing techniques, but the basic design is quite similar.

In constructing the cryostat, stainless steel was used wherever possible. Joints that do not have to be demountable were "heli-arc" welded and leak tested before assembly. This type of construction complicates modification or repair of the apparatus but almost completely eliminates troubles from vacuum leaks. Of the demountable joints, only two gasket seals and one soft-solder joint have to be vacuum tight.

The X-ray specimen cryostat is shown (in section) in figure 1. It is made up of two concentric Dewar vessels. Liquid helium is contained in the inner vessel (8) which has a hemicylindrical copper block (17) at the bottom. The specimen deposited on the plane, vertical surface of this block replaces the usual room temperature specimen. Beryllium windows (19) in the outer wall permit the X-ray beam to enter and leave the crvostat so that diffraction patterns of the material deposited on the surface can be obtained.

The helium vessel is surrounded by a liquid-nitrogen tank in the shape of a hollow cylinder (9) with copper radiation shields, (7) and (15), attached to it. The X-ray window in the radiation shield is covered with 0.00035 -in.-thick nickel foil (18) which shields the specimen from room-temperature surfaces and filters the $\mathrm{X}$-rav beam to eliminate copper $\mathrm{K} \beta$ radiation. Except for small openings for the specimen tube and optical windows, the helium vessel is entirely surrounded by surfaces which are at or near liquid-nitrogen temperature.

Vacuum spaces around the helium-cooled specimen 


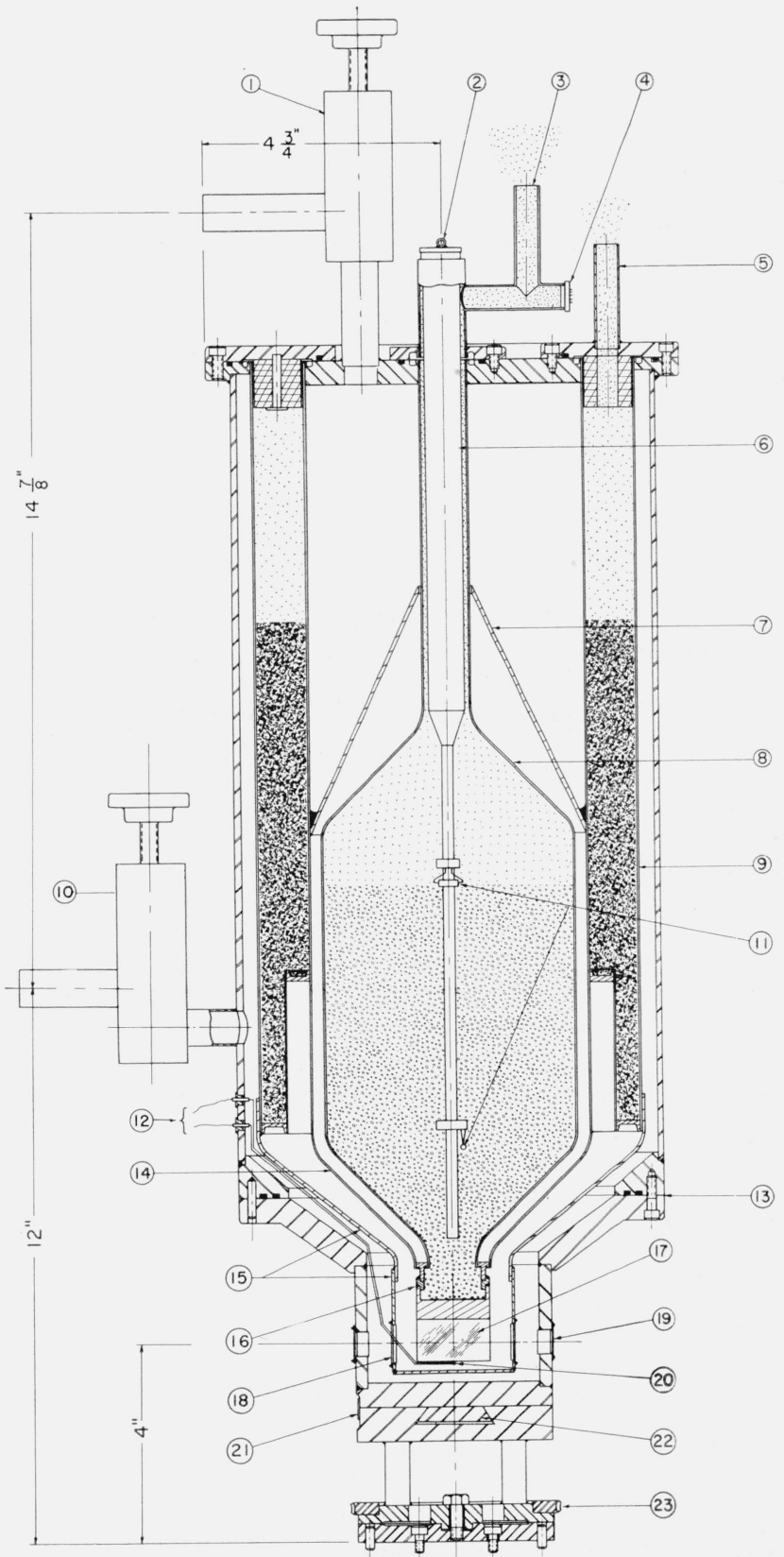

FIGURE 1. Section through the X-ray-diffraction cryostat.

1, Valve for evacuating helium Dewar; 2, helium fill-tube stopper; 3, helium vent; 4 , electrical connector for helium-level indicator; 5 , nitrogen vent or fill tube vent; 4, electrical connector for helium-level indicator; 5 , nitrogen vent or fill tube 9 , nitrogen vessel; 10 , valve for evacuating specimen chamber and nitrogen Dewar; 11, resistors for helium-level indicator $(2) ; 12$, thermocouple leads; 13 , demountable flange; 14 , inner-vacuum wall; 15 , lower radiation shield; 16 , brass ring and soft-solder joint; 17 , copper block; 18 , nickel foil X-ray windows 0.00035 in. thick 19 , beryllium X-ray windows; 20 , thermocouple junction; 21 , translational-ad justment vernier; 22 , translational-adjustment slide; 23 , rotational-adjustment worm gear.

block and around the outside of the nitrogen container are connected and are evacuated through a single valve (10) at the back of the apparatus. Another valve (1) is used to evacuate the space around the helium container. This space is separated from the specimen chamber by a wall (14) and seldom requires pumping. The wall probably accounts for as much as 75 percent of the heat transferred to the helium vessel once the specimen has been deposited, and reduces the operating time available on one filling to about $2 \mathrm{hr}$. However, separating the vacuum space around the helium vessel from that around the specimen prevents the sudden loss of helium and specimen that would result if the pressure in the specimen chamber should rise excessively during deposition or warmup of the sample.

X-ray windows (19) in the outer wall of the cryostat are covered with beryllium strips 0.025 in. thick. The two windows cover $85^{\circ}$ of arc each and permit measurements to be made in the angular range from $-2^{\circ}$ to $+168^{\circ} 2 \theta$. The beryllium strips are held in place with wire bands tightened by screws and are sealed to the stainless steel walls with Glyptal. The windows have not leaked during 6 months of use and have even survived a small azide explosion inside the cryostat.

A thermocouple (20) is used to measure the temperature of the copper block. For the alloys used (Ag plus 0.37 atomic percent $\mathrm{Au}$ versus $\mathrm{Au}$ plus 2.1 atomic percent $C_{0}$ ) the sensitivity is approximately $10 \mu \mathrm{v} /{ }^{\circ} \mathrm{K}$ at $4.2^{\circ} \mathrm{K}$. The junction is inserted in a well at the bottom edge of the specimen surface and the leads are cemented to the copper block to keep them at the temperature of the junction. The presence of gas in the specimen chamber affects heattransfer conditions, and if this precaution is not taken, temperature readings are pressure dependent.

The resistance of two carbon resistors (11) mounted in the helium vessel is recorded to indicate when liquid helium reaches the $1 / 4$-liter and the 2-liter levels. A current of about 50 ma flows through the two $15-\mathrm{ohm}$ resistors and the rate at which heat is carried away (by the gas in one case or the liquid in the other) determines the temperature and, hence, the resistance.

The outputs of the thermocouple and the liquidlevel gage are recorded continuously on a chart that is synchronized with the diffractometer chart.

Pressure measurements are made with a Philips cold-cathode ionization gage. Although the modification is not shown in the figures in this paper, the gage tube has recently been mounted on the cryostat itself so that pressure measurements can be made after the pumping manifold has been detached.

A slide (22) is provided for moving the cryostat on its base in a direction normal to the specimen surface in order to aline that surface with the axis of the goniometer. A worm gear (23) on the base is used to rotate the cryostat around a vertical axis to make the normal to the specimen surface bisect the angle between the incident and diffracted beams.

The dimensions have been chosen so that the slide can be removed from the base shown in figure 1 and inserted in another base that fits the North American Philips high-angle goniometer. However, that instrument has to be modified to operate with its axis vertical.

The lower chamber can be detached at the flange (13) to make the specimen block, thermocouple and lower radiation shield accessible for cleaning and 
repairs. The flange is also intended to permit the lower chamber to be replaced with a film cassette. If modification of the copper block is required, it can be removed by unsoldering the soft-solder joint (16). These provisions are intended to make it possible to modify the cryostat (e. g., for single-crystal work) if necessary .

The cryostat is shown mounted on the X-raydiffraction unit in figure 2. X-rays from the source slit (1) enter the cryostat through the beryllium windows (2). The diffracted beam enters the detector slit system (3). Its intensity is plotted by the recorder (4) against the diffraction angle, indicated on the protractor (5). Gaseous samples are introduced through the silica tube $(6)$. The specimen surface can be viewed through a glass window (7) in the outer wall. A system of pulleys and a counterweight balance the weight of the cryostat (68 lb) while leaving it free to rotate with the goniometer table. The supporting cables can be seen at the top of the cryostat.

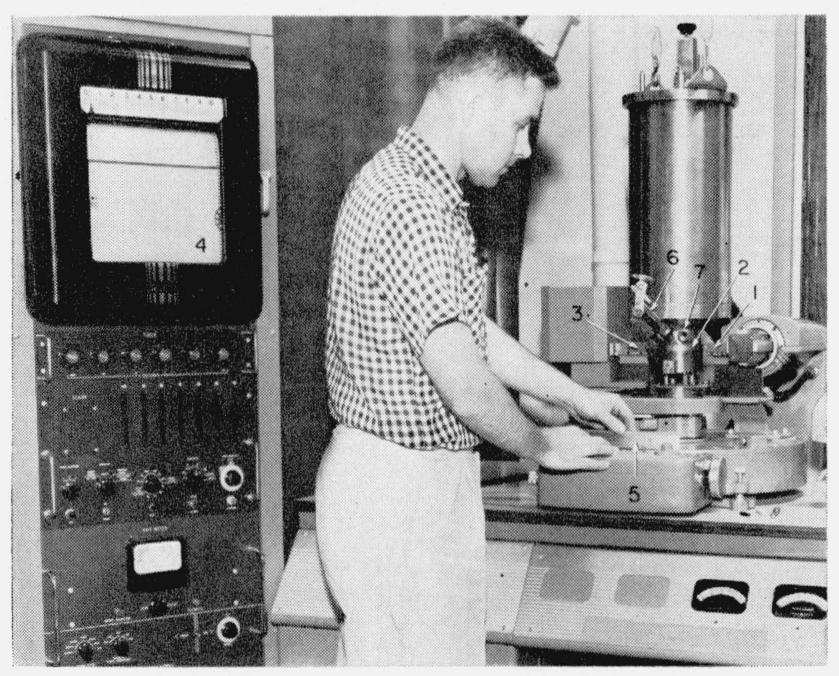

Figure 2. The cryostat mounted on the $X$-ray goniometer.

1 , X-ray source slit; 2 , beryllium window; 3 , detector slit; 4 , X-ray recorder; 5 . protractor; 6 , specimen discharge tube; 7 , window for viewing specimen surface (2).

\section{Operation}

The system used to evacuate the cryostat and deposit gaseous samples is shown schematically in figure 3. In preparing for a run at liquid-helium temperature, the specimen chamber is pumped down to $10^{-4} \mathrm{~mm}$ of $\mathrm{Hg}$ and liquid nitrogen is added to the cold trap. Within $15 \mathrm{~min}$ the pressure drops below $3 \times 10^{-5} \mathrm{~mm}$ of $\mathrm{Hg}$ and the cryostat is ready to be filled.

Before refrigerants are introduced, the gas to be deposited is usually pumped through the specimen chamber to simulate actual deposition of a sample on the helium-cooled surface. This aids in flushing the specimen manifold to remove contaminants, and permits the flow rate to be adjusted. After this adjustment the stopcock (fig. 3) between the capillary and the sample-gas input tube is closed so that the

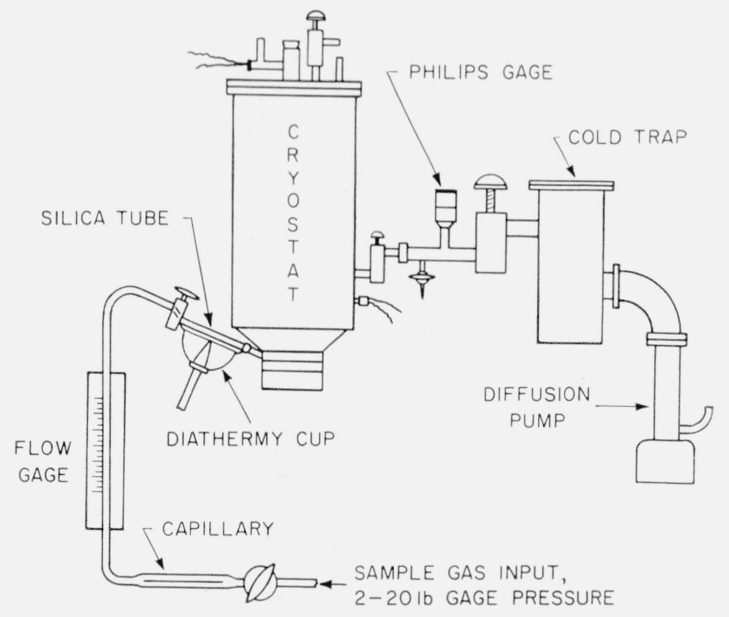

FIGURE 3. Gas train and pumping system used with the cryostat.

train up to that point is evacuated and gas does not collect in the tubing. The cryostat is then isolated from the pumping manifold by closing the valve and liquid nitrogen is introduced. Because of the cost of liquid helium, care must be taken to cool the apparatus to the temperature of liquid nitrogen before the helium is transferred. We prefer to fill the inner vessel to overflowing with liquid nitrogen and then syphon the liquid into the outer vessel. This method brings liquid nitrogen into contact with all the surfaces that must later be cooled by helium. After thorough precooling of the apparatus, approximately half a liter of helium is required to cool the inner Dewar to liquid helium temperature.

The procedure for producing free radicals and depositing them on a helium-cooled surface has been described by Broida and Pellam [10]. The system shown in figure 3 is suitable for most gases. but different equipment is sometimes used to deposit a liquid (through the vapor phase) or the gaseous products of a chemical reaction.

When the gas being deposited is to be dissociated to produce free radicals, it is passed through a silica tube in the rf field of a waveguide or diathermy cup. Power is provided by a $125-\mathrm{w}$ microwave-diathermy unit operating in the 2,400 - to $2,500-\mathrm{mc}$ band. With the power on, the gas flow is increased relative to the rate of condensation on the helium-cooled surface. When the flow is sufficient to give a pressure of 0.01 to $0.1 \mathrm{~mm}$ of $\mathrm{Hg}$ in the silica tube, a Tesla coil can be used to initiate the discharge. The gas (or free radicals) is allowed to deposit until the surface of the copper block loses its metallic appearance. Fifteen or twenty minutes is required to deposit the 0.001 to 0.005 -in. layer ordinarily used.

\section{Performance}

Once the sample has been deposited, obtaining $\mathrm{X}$-ray patterns is routine. Although 25 percent of the X-ray beam is lost in passing through the beryllium windows, this loss is partly compensated when the path inside the cryostat is evacuated. For 
copper radiation, the net loss is 15 percent. Intensities are adequate when the samples are crystalline. With the widest slits $\left(3^{\circ}\right.$ beam-divergence slit, medium-resolution detector Soller slit, and $0.2^{\circ}$ detector slit) counting rates considerably in excess of 10,000 cps are often attained. However, we do have evidence that crystallites deposited on the cold surface are often highly oriented so that the intensities of certain lines are greatly enhanced at the expense of others.

Helium is transferred from a 25-liter storage flask by means of a vacuum-insulated transfer syphon with a $1 / 8$-in. inner tube that extends 12 in. below the vacuum wall and reaches the bottom of the cryostat. With a pressure of $0.75 \mathrm{psi}$, less than $5 \mathrm{~min}$ is required to fill the helium Dewar to the 2-liter level. The Dewar will hold as much as $2 \frac{1}{2}$ liters of liquid helium, enough to last for $3 \mathrm{hr}$ under ideal conditions. Depositing samples always increases the boil-off rate and reduces the time at liquid-helium temperature to about $2 \mathrm{hr}$. This is sufficient for the measurements required. Much of the data is taken during normal warmup of the Dewar and additional helium capacity would only delay this important phase of the experiments.

If care is taken in alining the cryostat to allow for the thickness of the film deposited, there is no loss in the accuracy of interplanar spacings. Expansion curves are easily obtained by measuring the lattice constant of the specimen during warmup.

Resolving power of the diffractometer is not appreciably affected by the cryostat. The diffraction peak reproduced in figure 4 shows partial resolution of the $\mathrm{Cu} \mathrm{K} \alpha_{1}-\mathrm{K} \alpha_{2}$ doublet at $43.46^{\circ} 2 \theta$ where the separation is about $0.1^{\circ} 2 \theta$. Complete resolution of lines only $1.2^{\circ} 2 \theta$ apart is shown in figure 5 .

The apparatus has been useful not only in deter-

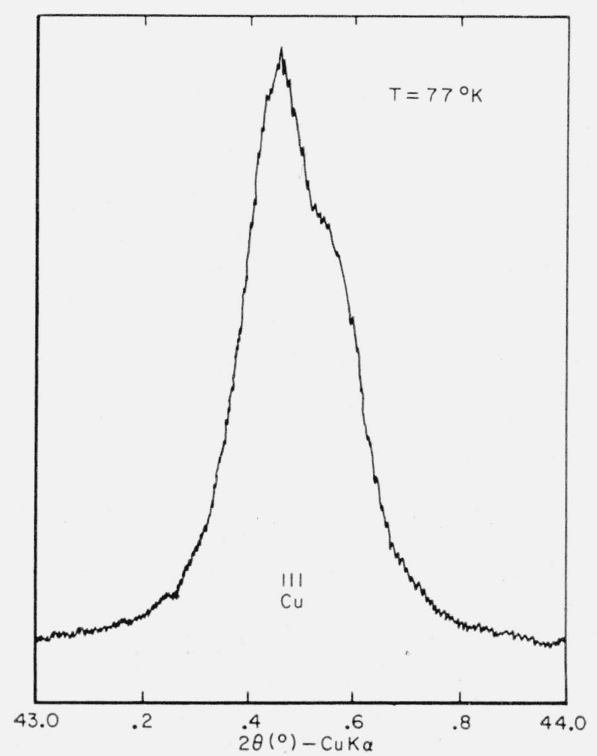

FIgURE 4. Diffraction peak showing partial resolution of the $\mathrm{CuK} \alpha_{1}-\mathrm{K} \alpha_{2}$ doublet.

The specimen was the copper block on which gaseous specimens are deposited.

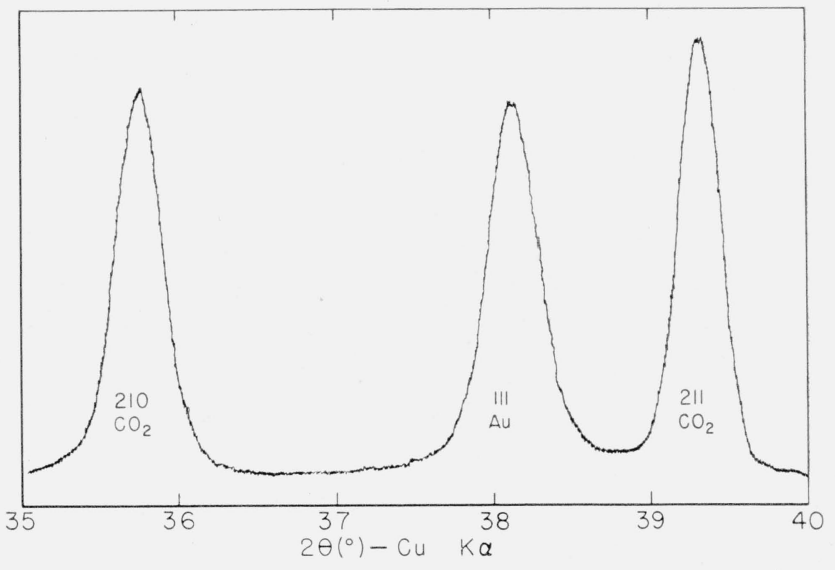

Figure 5. A portion of the diffraction pattern of annealed solid $\mathrm{CO}_{2}$ showing one peak due to the gold plating on the copper specimen block.

The pattern indicates the amount of instrumental line broadening to be expected.

mining the nature of the deposits originally frozen out at liquid-helium temperature, but in following the changes during warmup as well. The sudden crystallization of amorphous deposits with the evolution of considerable heat has been observed in several cases. Phase changes inferred from other types of measurements have been confirmed, and additional phases have been discovered. For example, X-ray patterns of the three phases of oxygen are shown in figure 6 along with a pattern of ozone

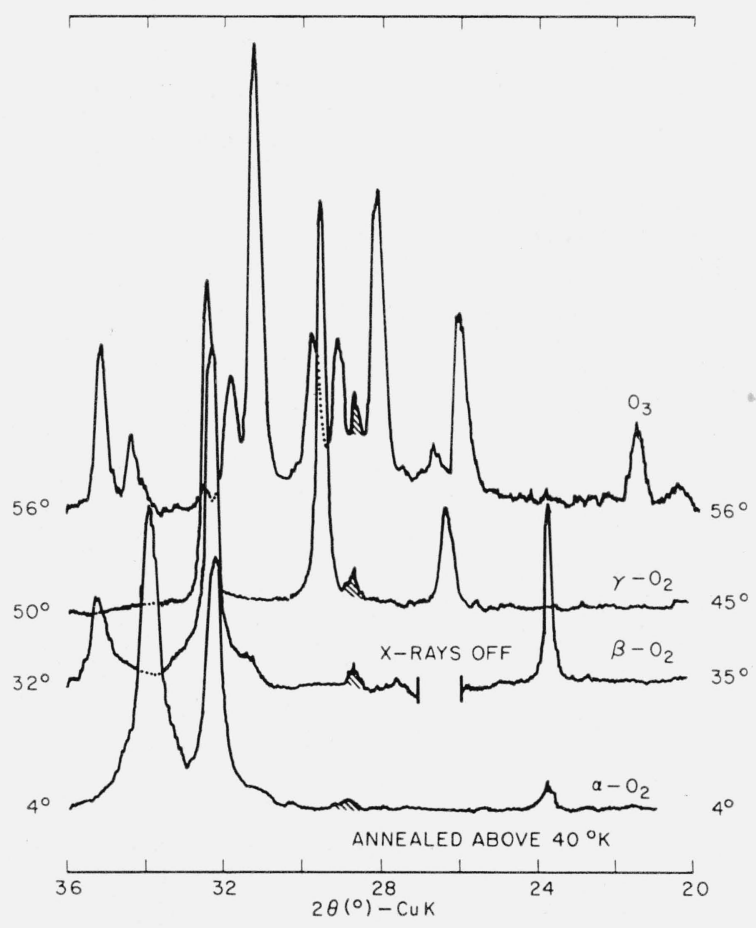

FIGURE 6. Diffraction patterns of solid ozone and the three phases of solid oxygen.

Numbers given in the margin are the approximate temperature in ${ }^{\circ} \mathrm{K}$ at the beginning and end of each diffractometer trace.

The line at $28.8^{\circ}(2 \theta)$ is due to contamination of the gold surface. 
prepared in a separate experiment by dissociating oxygen in a rf field. The dissociation products were deposited at $4.2^{\circ} \mathrm{K}$ and allowed to warm up until the residual oxygen sublimed at about $50^{\circ} \mathrm{K}$. A temperature of $56^{\circ} \mathrm{K}$ was maintained by pumping on nitrogen in the inner Dewar. These results will be reported in more detail in later papers dealing with specific materials.

The performance of the X-ray cryostat during 6 months of use has been entirely satisfactory. However, the natural warmup characteristics sometimes do not give enough time in a given temperature range to make the measurements required. Swenson and Stahl [8] have described a Dewar for maintaining temperatures between $4^{\circ}$ and $80^{\circ} \mathrm{K}$ by circulating helium gas cooled by liquid helium. Plans are being considered for adapting their technique or providing an auxiliary Dewar from which very small quantities of helium can be transferred at frequent intervals to arrest the warmup. Other modifications may become necessary if emphasis changes to the investigation of single crystals or orientation textures.

\section{References}

[1] M. and B. Ruhemann, Low temperature physies (Cambridge University Press, 1937

[2] H. S. Peiser, H. P. Rooksby, and A. J. C. Wilson, X-ray diffraction by polycrystalline materials (The Institute of Physics, London, 1955); (see chapter 10, Low temperature methods by E. G. Steward).

[3] A. G. Tweet, Phys. Rev. 93, 15 (1954).

[4] W. L. Gordon, C. H. Shaw, and J. G. Daunt, Phys. Rev. 96, 1444 (1954)

[5] Arthur Stephen Pavlovic, The design and application of a Weissenberg X-ray camera for studies neэr liquid helium temperature, The Pennsvlvania State University, Doctoral Dissertation Series, 1956, Publication No. 16725 .

[6] L. K. Jetter, C. J. MeHargue, R. O. Williams, and H. L. Yakel, Jr., Rev. Sci. Instr. 28, 1087 (1957).

[7] C. S. Barrett, Acta Cryst. 9, 671 (1956).

[8] C. A. Swenson and R. H. Stahl, Rev. Sci. Instr. 25, 608 (1954)

[9] W. H. Duerig and I. L. Mador, Rev. Sci. Instr. 23, 421 (1952).

[10] H. P. Broida and J. R. Pellam, Phys. Rev. 95, 845 (1954).

Washington, June 23, 1958. 